\title{
Improved Data Analysis and Reconstruction Methods for STEM-EDX Tomography
}

Zineb Saghi ${ }^{1,2,3}$, Joshua Einsle ${ }^{1,4}$, Roberts Blukis ${ }^{4}$, Ansis Strodahs ${ }^{1}$, Rowan Leary ${ }^{1}$, Pierre Burdet ${ }^{5}$, Richard Harrison ${ }^{4}$ and Paul Midgley ${ }^{1}$.

1. Department of Materials Science and Metallurgy, University of Cambridge, 27 Charles Babbage Road, Cambridge, CB3 OFS, UK.

2. Université Grenoble Alpes, F-38000 Grenoble, France.

3. CEA, LETI, MINATEC Campus, F-38054 Grenoble, France.

4. Department of Earth Sciences, University of Cambridge, Downing Street, Cambridge, CB2 3EQ, UK.

5. École polytechnique fédérale de Lausanne, SB-CIME, Station 12, 1015 Lausanne, Switzerland.

Energy dispersive X-ray (EDX) tomography is a valuable technique for the 3D compositional mapping of nanomaterials, but has been limited in the past by poor collection efficiency, long acquisition times and unreliable reconstructions [1,2]. With the recent development of high-brightness X-FEG guns and super-X EDX systems comprising four detectors, there has been a renewed interest in the technique and promising applications reported (e.g. [3-4]). While the acquisition of the EDX-STEM tilt series is still performed manually, progress has been made in data processing and algorithms for quantitative reconstruction. As an example, here, we have applied these advances to a Tazewell IIICD meteorite sample for the 3D chemical analysis of tetrataenite (FeNi) islands embedded in an Fe-rich matrix. A focused ion beam was used for the preparation of a needle-shaped sample, and a Fischione 2050 on-axis holder employed for the EDX-STEM tilt series acquisition using an FEI Osiris (S)TEM operating at 200kV. EDX-STEM maps (and the corresponding HAADF-STEM images) were recorded every $5^{\circ}$, with a dwell time of 30ms/pixel corresponding to 8min/map. Hyperspy, a python-based software for hyperspectral data processing [5], has been used for the batch-processing of the 4D dataset: the spectra were denoised using principal component analysis (PCA), and X-ray intensities extracted after background subtraction. The obtained tilt series of $\mathrm{Ni}$ and $\mathrm{Fe}$ maps were aligned using ImageJ, and exported to Matlab for tomographic reconstruction using compressed sensing (CS) [6]. This algorithm takes advantage of any prior knowledge about the sparsity of the object in a chosen transform domain and seeks a sparse solution that is consistent with the acquired data. CS has been shown to yield reliable reconstructions from a limited number of projections (e.g. [7-9]). Applied to the current dataset, sparsity in the image and gradient domains was promoted to generate Fe and Ni volumes, and the 3D (voxelwise) quantification was achieved in Hyperspy using the Cliff-Lorimer quantification method. Figure 1 (a-b) show the obtained volumes, displayed in weight percent. Figure (c-d) are slices through the volumes, highlighting the presence of Fe-rich secondary precipitates in the tetrataenite islands. These quantitative results were confirmed by atom probe tomography, performed on the same needle.

Multivariate statistical analysis methods, such as independent component analysis (ICA) [10] and nonnegative matrix factorization (NMF) [11], can also be applied to decompose 4D EDX datasets into a reduced number of component loadings, or phases, present in the material, thus speeding up both the data analysis and the reconstructions [12].

References:

[1] G. Möbus, R.D. Doole and B.J. Inkson, Ultramicroscopy 96 (2003), p. 433. 
[2] Z. Saghi et al. App. Phys. Lett. 91 (2007), 251906.

[3] K. Lepinay et al. Micron 47 (2013), p. 43.

[4] B. Goris et al. Nano Lett. 14 (2014), p. 3220.

[5] www.hyperspy.org

[6] R. Leary et al. Ultramicroscopy 131 (2013), p. 70.

[7] Z. Saghi et al., Nano Letters 11 (2011), p. 4666.

[8] O. Nicoletti et al. Nature $\mathbf{5 0 2}$ (2013), p. 80.

[9] S.M. Collins et al. ACS Photonics 2 (2015), p. 1628.

[10] D. Rossouw et al. Acta Mater. (2016), in press.

[11] S. Ikeshita et al., Micron 82 (2016), p.1.

[12] The research leading to these results has received funding from the European Research Council under the European Union's Seventh Framework Programme (FP/2007-2013)/ERC grant agreements 291522 - 3DIMAGE and 320750 - Nanopaleomagnetism. RKL acknowledges a Junior Research Fellowship at Clare College.
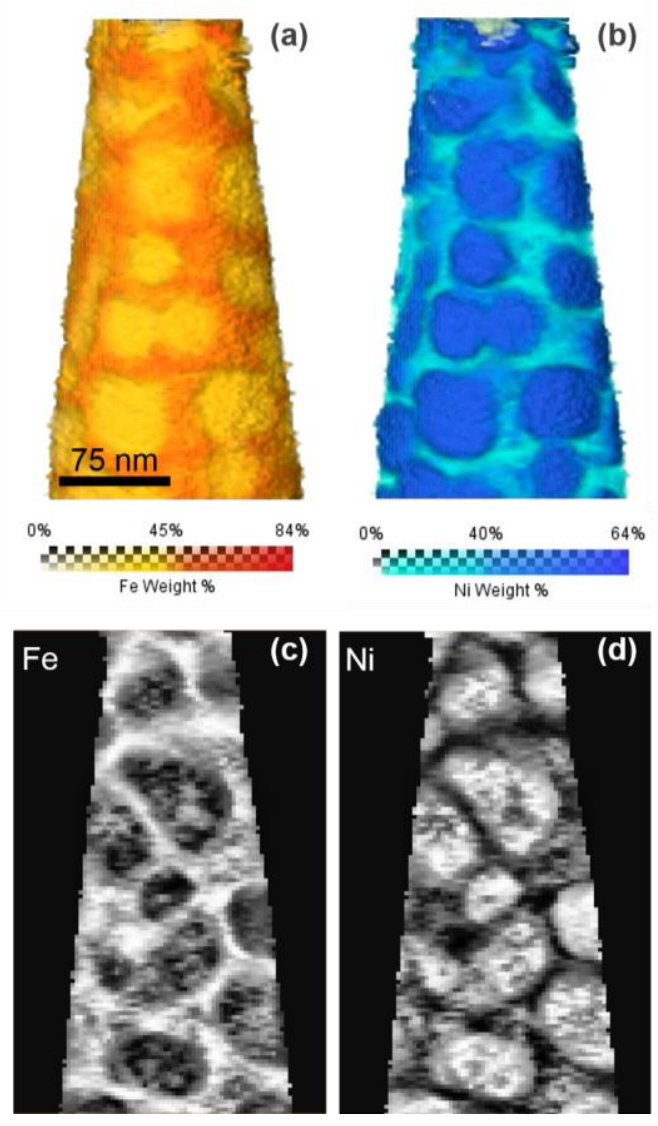

Figure 1. 3D chemical mapping of a Tazewell IIICD meteorite. (a-b) Voxel projection rendering of the $\mathrm{Fe}$ and Ni volumes, displayed in weight \%. (c-d) Orthoslices through the volumes showing the presence of Fe-rich secondary precipitates in the tetrataenite. 